EPJ Web of Conferences 75, 02003 (2014)

DOI: $10.1051 /$ epjconf/ 20147502003

(C) Owned by the authors, published by EDP Sciences, 2014

\title{
Surface magnetic domains dynamic in machined steel
}

\author{
D. Blažek ${ }^{1, a}$, M. Neslušan ${ }^{2}$ and D. Hrabovský ${ }^{3}$ \\ ${ }^{1}$ Nanotechnology Centre, VŠB-Technical University of Ostrava, 17. listopadu 15, 70833 Ostrava-Poruba, Czech Republic \\ ${ }^{2}$ Faculty of Mechanical Engineering, University of Žilina, Univerzitná 1, 01026 Žilina, Slovakia \\ ${ }^{3}$ RMTVC, VŠB-Technical University of Ostrava, 17. listopadu 15, 70833 Ostrava-Poruba, Czech Republic
}

\begin{abstract}
This contribution deals with an observation of the magnetic dynamic of different types of the machined surface of bearing steel. The Bakhausen noise (BN) measurements are presented here as commonly introduced in industry for quality control due to the extremely sensitivity of the magnetic domains wall dynamics to the microstructure of material. The results of magneto-optical measurements are presented with the goal to explain the observed BN anisotropy. It is shown that BN anisotropy is associated with uniaxal magnetic anisotropy introduced by hard milling which causes the principally different magnetic reversals processes in orthogonal directions.
\end{abstract}

\section{Introduction}

Steels are heat treated and machined to impart the desired functional properties. Physical properties and microstructure of the material surfaces are strongly affected by these technological processes. High hardness and magnetic response are exclusive characteristics in the sense that decreasing hardness is associated with rising coercivity and Barkhausen noise $(\mathrm{BN})$ [1,2]. The residual stress is reflected in anisotropic magnetic properties.

Here we report on the detailed study of $\mathrm{BN}$ and magneto-optically measured hysteresis curves of machined surfaces on bearing steels. Comparing these results the nature of ongoing processes is revealed.

\section{Experimental material and conditions}

The experimental study was carried out on the OVAKO through-hardened bearing steel $100 \mathrm{Cr} 6$ (according to EN 10027-1), see Table 1. for chemical composition. These steels exhibit high fatigue and wear resistance, good machinability, high hardness and good dimensional stability. The hardness up to $65 \mathrm{HRC}$ can be achieved through martensitic hardening. The mechanical and magnetic properties of the material can be affected by tempering. Two bulk states of material were used, softened to the $61 \mathrm{HRC}$ and to the $45 \mathrm{HRC}$ hardness.

Table 1. Chemical composition of the studied material

\begin{tabular}{|c|c|c|c|c|c|c|}
\hline $\begin{array}{c}\text { Componen } \\
\mathbf{t}\end{array}$ & $\mathbf{C r}$ & $\mathbf{C}$ & $\mathbf{M n}$ & $\mathbf{S i}$ & $\mathbf{S}$ & $\mathbf{F e}$ \\
\hline wt. \% & 1.50 & 0.97 & 0.35 & 0.25 & $<0.015$ & balan. \\
\hline
\end{tabular}

\footnotetext{
${ }^{\mathrm{a}}$ Corresponding author: dblazek@gmail.com
}

The studied surfaces of material were prepared by the wet grinding and hard milling operation. The grinding surface (conditions: BPH 20, grinding wheel A99 60J9V, $\mathrm{v}_{\mathrm{c}}=25 \mathrm{~m} \cdot \min ^{-1}, \mathrm{v}_{\mathrm{f}}=8 \mathrm{~m} \cdot \min ^{-1}, \mathrm{a}_{\mathrm{p}}=0,01 \mathrm{~mm}$, wet Ecocool 3\%, 3 spark out passes) is mainly thermally loaded which causes surface hardness decrease, some phase transitions in heat affected zone (HAZ) and slightly anisotropic residual stress. Hard milling (conditions: FA4 AV, dry, cutting inserts made of cemented carbides R300-1240E-PM, tool holder R300-050Q22-12M $262489, \mathrm{a}_{\mathrm{p}}=0,25 \mathrm{~mm}, \mathrm{v}_{\mathrm{f}}=112 \mathrm{~mm} \cdot \mathrm{min}^{-1}, \mathrm{n}=500 \mathrm{~min}^{-1}$ ) initiate high temperatures and considerable hydrostatic pressure at contact with cutting edge. The temperature in the tool-work piece interface exceeds Currie temperature. Very anisotropic huge tensile residual stresses remain in surface layer after milling as a result of plastic deformation.

Throughout the article, the tangential direction (t) is considered to be parallel to the cutting direction and perpendicular direction (p) is the normal one. Thus defined directions correspond to the principal axes of the tensor of residual stress. For measured principal stresses see Table 2 .

Table 2. Surface residual stresses after machining measured by X-ray diffraction.

\begin{tabular}{|c|c|c|c|}
\hline Method & $\begin{array}{c}\text { Wet. } \\
\text { grinding }\end{array}$ & $\begin{array}{c}\text { Dry } \\
\text { grinding }\end{array}$ & $\begin{array}{c}\text { Hard } \\
\text { milling }\end{array}$ \\
\hline$\sigma_{\mathrm{t}}[\mathrm{MPa}]$ & -147 & 332 & 528 \\
\hline$\sigma_{\mathrm{p}}[\mathrm{MPa}]$ & -320 & 192 & 278 \\
\hline
\end{tabular}




\section{Experimental methods}

The residual stresses presented in Table 2. were measured via X-ray diffraction technique performed on the X'Pert PRO diffractometer ( $\{211\} \alpha-\mathrm{Fe}, \mathrm{CrK} \alpha, \mathrm{X}^{\prime}$ Pert PRO).

\subsection{Magneto-optical Kerr effect method (MOKE)}

In order to investigate the magnetization reversal processes, both components of the surface magnetization parallel and perpendicular to the in-plane applied field were measured as a function of the magnetic field intensity. The experimental set up is shown in Figure 1.

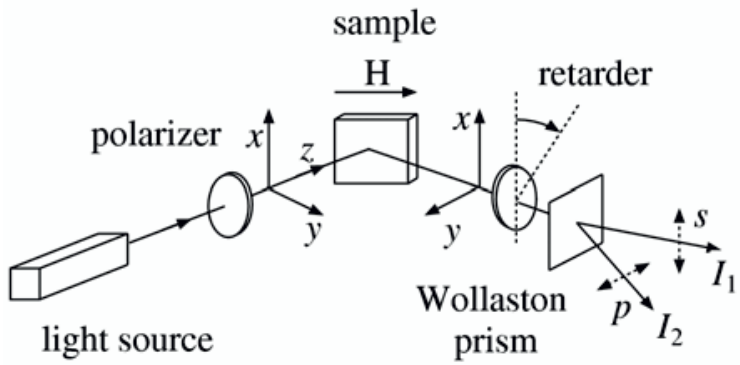

Figure 1. The MOKE experimental setup.

A laser beam with the wavelength $650 \mathrm{~nm}$ and power $5 \mathrm{~mW}$ amplitude modulated at the frequency $50 \mathrm{kHz}$ was used. The incident angle was $45 \mathrm{deg}$ and the MOKE was measured at the reflection angle $45 \mathrm{deg}$ (zero order of diffraction). The component of magnetization parallel to the incident plane was measured as a consequence of the linear dependence of the conversion reflection coefficients $r_{\mathrm{sp}}=r_{\mathrm{ps}}$ on the longitudinal magnetization [3]. The light penetration depth is less than100nm.

The sample was immovable attached to the magnetic circuit, and rotated together in order to measure both components of magnetization. In the following, the component of magnetization measured when the field is parallel to the incident plane will be named longitudinal and when the field is perpendicular to the incident plane it will be named transversal. At each sample four hysteresis curves (tangential longitudinal and transversal and perpendicular long. and trans.) was measured in the same experimental conditions.

\subsection{The Barkhausen noise (BN) measurement}

The BN was carried out with Microscan 500 device with the standard probe photographed in the Figure 2. The affected area is about $1 \mathrm{~cm}^{2}$. The magnetizing coil was powered by harmonic voltage with amplitude $10 \mathrm{~V}$ in the frequency range $10 \mathrm{~Hz}-1000 \mathrm{~Hz}$. The magnetic field penetration depth depends on the frequency, still large compared to the thickness of altered surface. Most of the here presented results were measured at the frequency $100 \mathrm{~Hz}$ due to the highest signal to noise ratio. The sampling frequency was $2.5 \mathrm{MHz}$. The $\mathrm{BN}$ magnitude was calculated from moving RMS value (with period of 200 measured points) adjusted for noise. Depending on the probe orientation the $\mathrm{BN}$ in tangential or perpendicular direction is obtained.

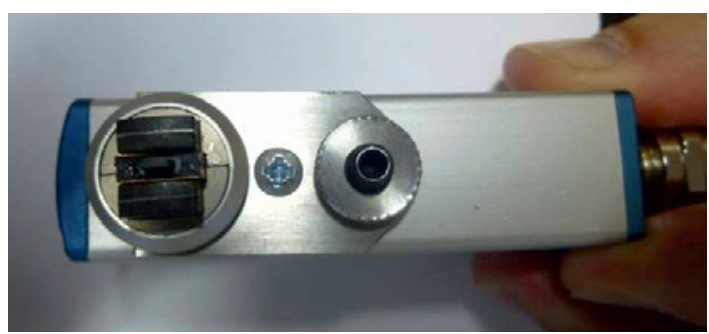

Figure 2. The probe for measurement of the surface BN.

\section{Experimental results}

A complete set of measurements on four samples was performed. The wet grinded and hard milled surfaces were prepared on the $61 \mathrm{HRC}$ and $45 \mathrm{HRC}$ materials. These surfaces captured by stereomicroscope are presented in Figure 3. The roughness of the surfaces is of the order of $10 \mu \mathrm{m}$ with evident periodicity in the perpendicular direction.
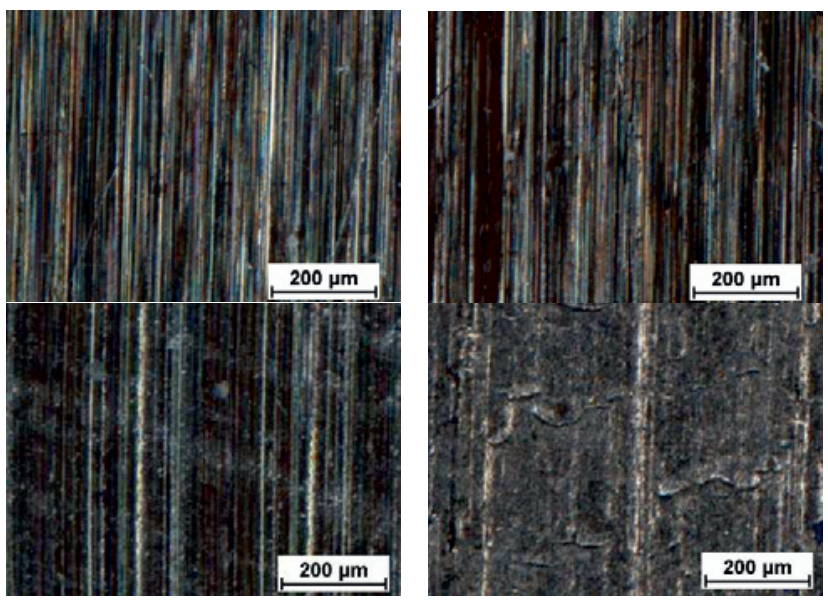

Figure 3. The examined surfaces. The wet grinded in upper row and hard milled in bottom row. The 61 HRC material left and 45 HRC right.

The measured BN values from all samples in both directions are presented in Table 3. An undetectable anisotropy on the grinded samples is due to the big noise magnitude about $25 \mathrm{mV}$. Presented values are adjusted from noise. It should be noted that obtained $\mathrm{BN}$ values, signal to noise ratio and anisotropy ratio significantly depend on the frequency.

Table 3. BN values measured at $100 \mathrm{~Hz}$ (mean BN magnitude during one period).

\begin{tabular}{|c|c|c|c|}
\hline Sample & $\begin{array}{c}\text { Tangential } \\
\text { direction }\end{array}$ & $\begin{array}{c}\text { Perpendicular } \\
\text { direction }\end{array}$ & $\begin{array}{c}\text { Anisotropy } \\
\text { ratio }\end{array}$ \\
\hline $\begin{array}{c}61 \mathrm{HRC} \\
\text { grinded }\end{array}$ & $30 \mathrm{mV}$ & $30 \mathrm{mV}$ & $1: 1$ \\
\hline $\begin{array}{c}61 \mathrm{HRC} \\
\text { milled }\end{array}$ & $200 \mathrm{mV}$ & $46 \mathrm{mV}$ & $4: 1$ \\
\hline $\begin{array}{c}45 \mathrm{HRC} \\
\text { grinded }\end{array}$ & $129 \mathrm{mV}$ & $129 \mathrm{mV}$ & $1: 1$ \\
\hline $\begin{array}{c}45 \mathrm{HRC} \\
\text { milled }\end{array}$ & $251 \mathrm{mV}$ & $46 \mathrm{mV}$ & $5: 1$ \\
\hline
\end{tabular}




\subsection{The wet grinded surfaces}

The measured $\mathrm{BN}$ signals consist from the sequence of pulses which comes from collective detection of many moving domain walls. Due to the high magnetization rate the signal cannot distinguish the single jumps nor the avalanches. The interpretation of the data must be fully phenomenological based on the magnitude of $\mathrm{BN}$ which reflects the domain dynamics.[4]

The BN signals measured on wet grinded surfaces are presented in Figure 4. These results are consistent with the fact that the low hardness steels usually emit strong pulses with corresponding high level of BN.[1]
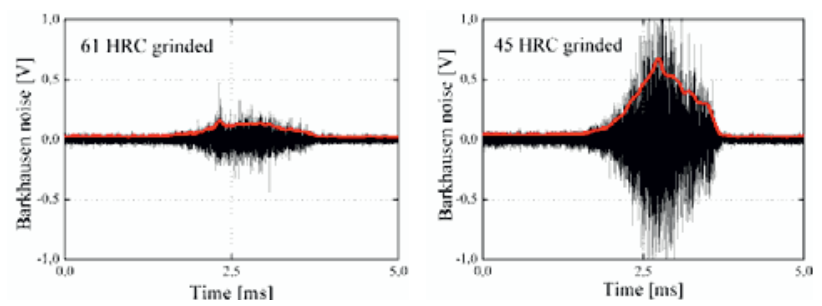

Figure 4. The measured $\mathrm{BN}$ signals on the wet grinded surfaces. The $61 \mathrm{HRC}$ material left and $45 \mathrm{HRC}$ right.

The isotropic $\mathrm{BN}$ could lead to the idea that measured hysteresis curves $(\mathrm{HC})$ are the same in all directions. MOKE measurements presented in Figure 5. show that this is not the case. These results seem like the $\mathrm{HC}$ of the system with the presence of the uniaxial easy axis parallel to the cutting direction, especially in the case of grinded 61HRC material.
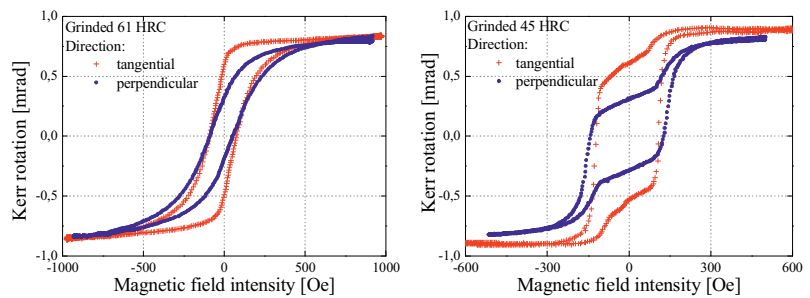

Figure 5. The measured MOKE hysteresis curves on the wet grinded surfaces. The $61 \mathrm{HRC}$ material left and $45 \mathrm{HRC}$ right.

Relying on the simulations based on the StonerWohlfarth model (SW), the uniaxial anisotropy affects not only the slope of HC (the effective permeability) but also the coercivity. We consider the same size coercive field measured in both directions to be contra-indicative to the inherence of uniaxial easy axes. This implies the necessity to include additional effects, such as the demagnetizing field due to the surface relief for example.

\subsection{The hard milled surfaces}

The BN signals of the hard milled $45 \mathrm{HRC}$ surface are in Figure 6. The strong anisotropy of this $\mathrm{BN}$ is obvious. The $\mathrm{BN}$ magnitude in tangential direction is two times higher, and magnitude in perpendicular direction is one third compared with the $\mathrm{BN}$ of grinded surface.
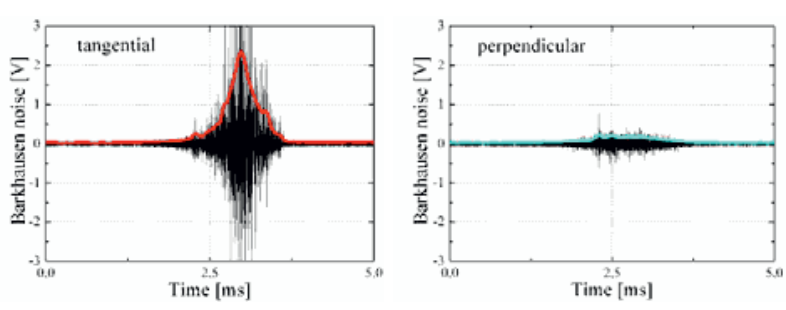

Figure 6. The BN signals on the 45 HRC hard milled surface. The tangential direction left and perpendicular right.

The BN signals of the hard milled 61 HRC surface are of the greater magnitude in both directions compared to the BN of grinded $61 \mathrm{HRC}$ surface. Looking at Figure 7., these signals are very similar to that measured on milled 45 HRC surface. The only difference lies in the height and rounded base of the burst measured in tangential direction.
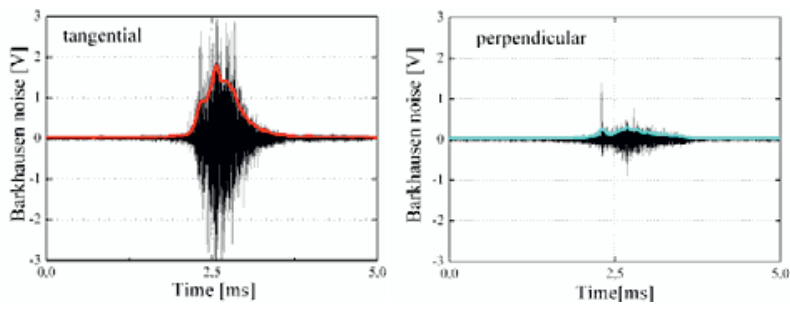

Figure 7. The BN signals on the 61 HRC hard milled surface. The tangential direction left and perpendicular right.

The strong anisotropy of $\mathrm{BN}$ signals measured on hard milled surfaces is associated with the different $\mathrm{HC}$ measured in orthogonal directions by MOKE as can be seen in Figure 8.
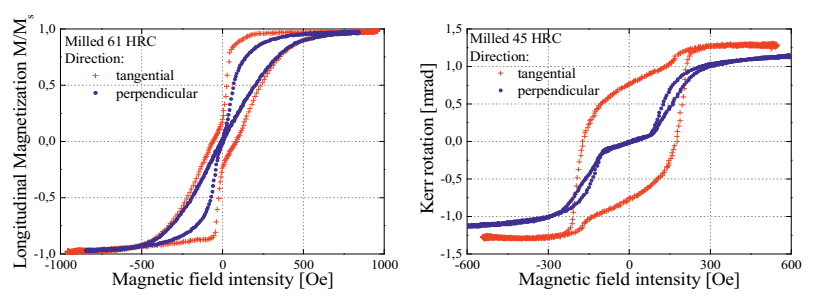

Figure 8. The measured MOKE hysteresis curves on the hard milled surfaces. The $61 \mathrm{HRC}$ material left and $45 \mathrm{HRC}$ right.

It is obvious, that $\mathrm{HC}$ measured in the tangential direction widened while the $\mathrm{HC}$ measured in perpendicular direction extremely narrowed to the zero coercivity and remanence. This behaviour may be explained by the strong uniaxial easy axis in the tangential direction.

What is interesting, the classical shape of surface HC of the wet grinded 61 HRC material considerably changed after hard milling, and the magnetisation reversal of the milled material tends to occur in two distinct steps. These changes are probably related with the softening of 61HRC material in heat affected zone during the milling due to the achieved temperatures and associated structure alterations. This idea needs to be confirmed by further structural analysis. 
In the next Figure 9., there are the obtained BN bursts from all examined samples. It is obvious that the bursts measured at hard milled surfaces are much more similar to each other than $\mathrm{BN}$ signals measured on the wet grinded samples.
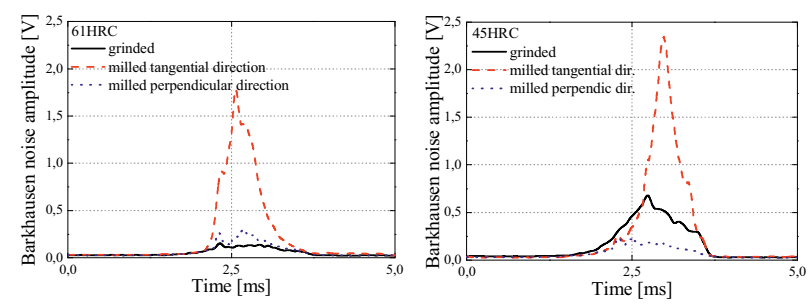

Figure 9. The BN signals on the $45 \mathrm{HRC}$ hard milled surface.

\subsection{The magnetic reversal of $45 \mathrm{HRC}$ hard milled}

The results presented in the previous subsection clearly demonstrate that magnetic reversals processes are principally different depending on the direction of the applied magnetic field. In order to clarify the nature of these processes, both longitudinal and transversal components of magnetization were measured by MOKE setup on all samples. The biggest difference was obtained on the $45 \mathrm{HRC}$ hard milled sample while negligible differences were obtained on wet grinded surfaces. .
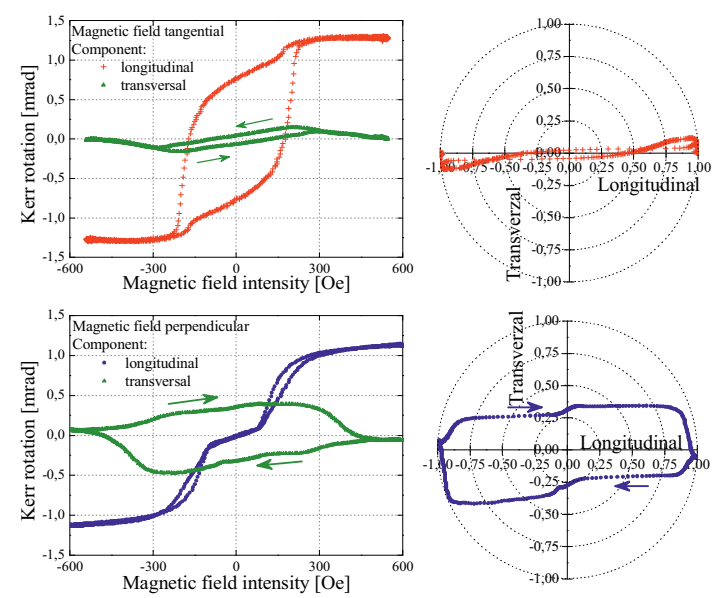

Figure 10. The magnetization reversal process measured by MOKE. Both components of magnetization, longitudinal and transversal, are presented as a function of magnetic field intensity (left) and in polar coordinates (right). The magnetic field is applied in tangential direction (up row) and perpendicular direction (bottom row).

The results presented in Figure 10. show that the magnetic reversals process in the case of the tangential magnetic field consists of the switch by $180^{\circ}$ with negligible transversal component. During this continuous nucleation and growth of opposite oriented domains the sample passes through the demagnetized state.

In the case of perpendicular magnetic field, the reversal process consists of the coherent rotation of magnetization followed by two-step $90^{\circ}$ switches and completed by coherent rotation. The magnetization never drops below $25 \%$ of the saturated value, which is the reason why the sample cannot be demagnetized by alternating perpendicular field. These results are consistent with these obtained on epitaxial Fe film [5].
Seen in this light, the very anisotropic $\mathrm{BN}$ can be explained by the consideration of the $90^{\circ}$ domain walls being bad sources of $\mathrm{BN}$ in contrast to $180^{\circ}$ being significant sources of $\mathrm{BN}$.

\section{Conclusions}

The magnetization reversal of the machined surfaces on the bearing steel OVAKO 100Cr6 has been studied by MOKE and Barkhausen noise emissions. The observation of both magnetization components allows us to investigate the reversal process and reveal the nature of measured BN. We demonstrate that MOKE method can be successfully used on polycrystalline surface with roughness of the order of $10 \mu \mathrm{m}$.

The measured results shown that wet grinded surfaces reveals isotropic $\mathrm{BN}$ but different $\mathrm{HC}$ in orthogonal directions. This HC anisotropy could not be elucidated by uniaxial magnetic anisotropy.

Contrariwise, in the case of hard milled surfaces the uniaxial magnetic anisotropy parallel to the cutting direction is an important controlling factor that affects the nature of magnetic reversal processes. From transversal MOKE measurements, it is obvious that uniaxial anisotropy promotes domain nucleation and magnetic reversal in one step if the magnetic field was applied in tangential direction and contrary it supports the rotation of magnetization in the plane combined with two-step switching if the magnetic field was perpendicular to the machining direction. The reversal process in tangential direction is performed using $180^{\circ}$ domain walls while in perpendicular direction it is using $90^{\circ}$ domain walls.

The very anisotropic $\mathrm{BN}$ can be explained by the assumption of the $90^{\circ}$ domain walls being bad sources of $\mathrm{BN}$ in contrast to $180^{\circ}$ being significant sources of $\mathrm{BN}$. As a result of mentioned factors, the stronger uniaxial anisotropy is reflected in very anisotropic BN with measured ratio up to 5:1 in orthogonal directions.

\section{Acknowledgments}

The research is supported by IT4Innovations Centre of Excellence project, reg. no. CZ.1.05/1.1.00/02.0070, Regional Centre of Excellence reg. no. CZ.1.05/2.1.00/01.0040, New creative teams in priorities of scientific research. (CZ.1.07/2.3.00/30.0055), and by the Grant Agency of the Czech Rep. (no. P205/11/2137).

The authors are grateful to Dr. M. Bukovina, University of Žilina, for photos from stereomicroscope.

\section{References}

1. M. Vashista, S.Paul, JMMM 323 , 2579-2584 (2011)

2. V. Moorthy, B.A. Shaw, P. Hopkins, JMMM 299, 362-375 (2006)

3. K. Postava et all., JMMM 172, 199-208 (1997)

4. G. Durin, S. Zapperi, The Science of Hysteresis II, p.181-267 (2005)

5. Q. Zhan, S. Vandezande, K. Temst, Ch. V. Haesendonck, Phys. Rev. B 80, 094416 (2009) 